\title{
Swift and Suzaku observations of spectral evolution in the FRED type GRBs
}

\author{
MAKOTO TASHIRO* \\ Department of Physics, Saitama University \\ E-mail: tashiro@phy.saitama-u.ac.jp
}

\section{Haruka Ueno; Jun'ichi Enomoto}

Department of Physics, Saitama University

\begin{abstract}
The energy dependence in light curves of gamma-ray bursts (GRBs) is a probe to study the underlying radiation mechanism. In particular, spectral evolution in the decay phase is expected to reflect the cooling process of accelerated electrons. Norris et al. [1] systematically examined asymmetric pulses in the prompt emissions of GRBs and showed that the pulse widths have the energy dependence that is well approximated with a power-law with the energy index of -0.41 in average. Although they did not particularly mention about the decay phase, their result strongly suggests a universal radiation/cooling mechanism in the emission region of GRBs. In previous study with Suzaku/WAM[2] , we sampled 6 bright GRBs; 7 well isolated pulses that showed no power-law decay but exhibiting exponential-decay (FRED) were detected in total, and found that the time constants evaluated for each energy band exhibited a power-law type energy dependence with the energy index of -0.3 to -0.5 . Now our next step would be to investigate the radiation process in the GRB prompt emissions in wider energy bands beyond Suzaku/WAM. Here we report the results of our study of the three bright GRBs (GRB 060117, GRB 070917, GRB 080413B) that showed the FRED and were observed with both Swift/BAT and Suzaku/WAM. All of their exponential decays exhibit similar power-law type energy dependence. The distribution of the energy indices is consistent with the FREDs that were observed with WAM, as reported in [2]. Our detailed time-resolved spectral study reveals that the spectra of all the three FREDs are well reproduced with the Band GRB functions with decreasing turnover energy. In particular, the time evolution of two of the three FREDs are consistent with those expected in the fast synchrotron-cooling regime [3].
\end{abstract}

Swift: 10 Years of Discovery

2-5 December 2014

La Sapienza University, Rome, Italy

\footnotetext{
* Speaker.

${ }^{\dagger}$ present address: Japan Aerospace Exploration Agency
} 


\section{Introduction.}

The spectrum of GRB prompt emission has long been described with a power law with an exponentially cut-off, or a smoothly-connected broken power-law (GRBM [4]). They are assumed to be produced by partially-cooled optically thin plasma electrons via synchrotron or inverseCompton radiation on the shock fronts in jets. In addition to that, an optically thick component is proposed to describe the observed curvature in the sub-MeV band (e.g. [5]). As well as wideband spectroscopy up to $\mathrm{GeV}$ band, spectral time evolution has become a key aspect to resolve physics in the prompt emission region (e.g. [6]; [2]; [7]; [8]). In order to conduct the luminosityspectrum evolution study with large effective areas in sub-MeV band, we here employed the Swift/ Burst Alert Telescope (BAT) [9] and the Suzaku/ Wide-band All-sky Monitor (WAM) [10].

\section{Fast-Rise-Exponential-Decay (FRED) light curve}

In general, time scales of brightening and dimming are determined with geometrical parameters, such as size and bulk motion of the emission plasma, and by the electron acceleration/deceleration mechanisms. Among them the longest time scale dominates the observed light curve in the rise or decay phase. In order to reduce the number of parameters that affect the time evolution, we focus on the decay phase of fast-rise-and-exponential-decay (FRED) shaped light curves. The fast rise implies that the time scale of geometrical variation is sufficiently short that it does not appear in the longer decaying phases. The longer time scale in decay is thus expected to reflect the state evolution of the emission region, including the radiation cooling process. The exponential decay must be a result of the radiation cooling process, whereas the geometrical effect, such as expansion and/or deceleration of the radiation surface, should be observed as a power-law type decay in the energy bands that exhibit a power-law like spectrum.

In 2012, we reported 7 FRED pulses observed with WAM, and found a common energy dependence of exponential decay time-constants among them [2]. In this contribution, we add 3 samples observed with both Suzaku/WAM and Swift/BAT simultaneously. With the wide-band time-evolution study, we extend investigation on the radiation process of the long GRBs.

We selected FRED GRBs observed both with WAM and BAT by using the following criteria: (1) bright ( $>1 \mathrm{c} / \mathrm{s} /$ BAT-detector) at peak, (2) asymmetric (Fast rise but slow in decay), (3) no overlapping of pulses in the decay phase (to avoid picking up other acceleration or radiation processes). Thus we selected 3 FRED peaks in addition to the reported 7 FREDs by WAM. All the quoted errors below are at statistical $90 \%$ confidence level.

\section{Energy resolved light curves}

Here we employed the light curves of BAT and WAM with each time bin of $0.5 \mathrm{~s}$, in order to evaluate the trend of spectral variability. We confirmed similar properties as those reported in our previous WAM study [2]; (1) every decay is exponential (power-law decay is rejected), and (2) higher the energy band of the light curves is, shorter the time constant is. 

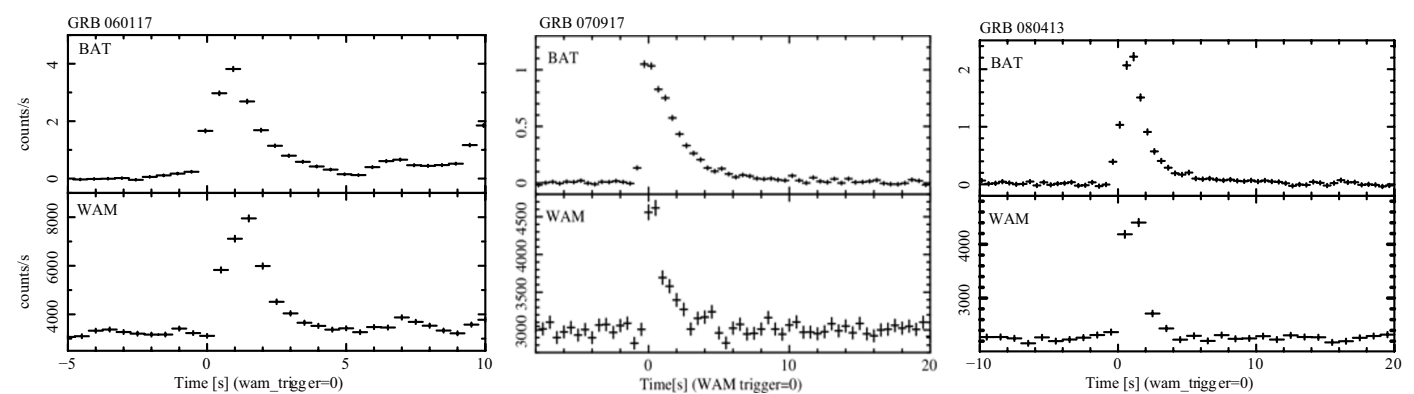

Figure 1: The FRED-type GRB light curves observed both with Swift/BAT (top) and Suzaku/WAM (bottom) from GRB 060117 (left), GRB 070917 (middle) and GRB 080413 (right).

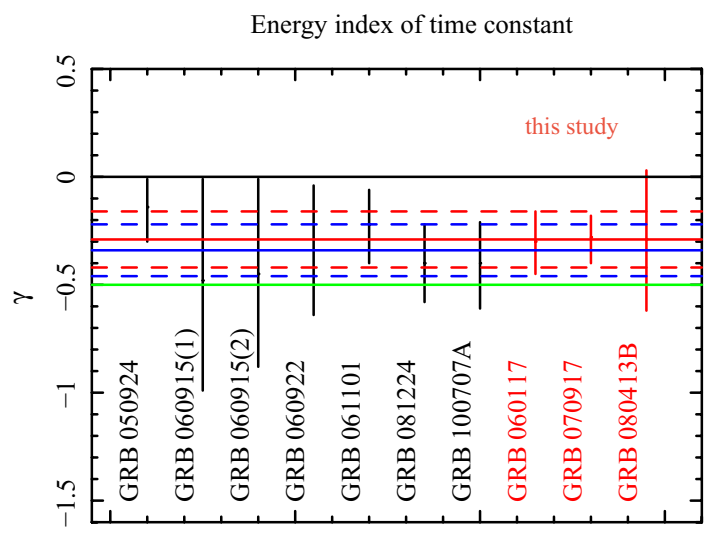

Figure 2: The derived energy indices $(\gamma)$ of time constants of exponential decays in FREDs. The 3 events on the right (red ones) are of this study, while the other 7 are taken from Tashiro et al. [2]. The black and green solid lines indicate the value of $\gamma$ expected in cases of "no spectral variation" and of "a simple synchrotron cooling", respectively. The red and blue solid lines are the average values for the 3 BAT+WAM $\left(\gamma_{\text {ave. }}^{B W}\right)$ and 7 WAM-only events $\left(\gamma_{\text {ave. }}^{W}\right)$, respectively, and the dashed lines in each color indicate their $90 \%$ confidence region.

In order to evaluate the trend quantitatively, we evaluate the decay time constant derived from the light curve in each energy band with a power-law function of energy of,

$$
\tau(E)=N E^{\gamma}
$$

where $\tau$, and $N$ denote decay time constant at energy $E$ and the factor of proportionality, respectively. Figure 2 summarizes the obtained best-fit value of $\gamma$ with the $90 \%$ confidence level in comparison with those reported in [2]. Derived $\gamma$ tightly distributes around the average value of BAT+WAM reported in this study $\gamma_{\text {ave. }}^{B W}=-0.29$, and is consistent with the distribution derived by Tashiro et al. [2] of $\gamma_{\text {ave. }}^{W}=-0.34$. Although 6 out of 10 energy indices $\gamma$ are consistent with the value expected in simple synchrotron-cooling regime $(\gamma=-0.5)$ [11, the rest 4 reject the value with the confidence level of $90 \%$. The discrepancy implies that they are not subject to a simple (slow) cooling regime and/or may require additional component(s). 


\section{Time resolved spectrum}

The newly analyzed 3 GRBs are localized by Swift/BAT and the incident angles to WAM are accurately determined to generate reliable energy response matrices of WAM. We investigate timeresolved spectra to examine the emission mechanism models - GRBM, or a blackbody radiation with an additional power-law shape emission component (BBPL) (e.g. [6]).

\subsection{GRB 060117 and GRB 070917 - fast-cooling synchrotron}

The 1-s time-resolved spectra from the decay phase in GRB 060116 and GRB 070917 exhibit a statistically significant preference for GRBM over BBPL. Then we evaluate the spectral softening with the turn-over energy $E_{0}$, employing the time index $\eta$ as,

$$
E_{0}(t) \propto t^{\eta} .
$$

We assumed a decreasing normalization of GRBM in proportion to $t^{-1 / 4}$, according to the fast cooling regime [3], and tied the first and second spectral indices. Table 1 lists the derived evolution of the turnover energy $E_{0}$ and $\chi^{2} /$ d.o.f. together with the derived time indices $\eta=-1.13_{-0.21}^{+0.23}$ and $\eta=-1.48_{-0.25}^{+0.26}$ of GRB 060116 and GRB 070917, respectively. These values agree with the expected time index in the fast-cooling regime $(\eta=-3 / 2)$ with $90 \%$ and $95 \%$ confidence levels, respectively.

GRB 060117

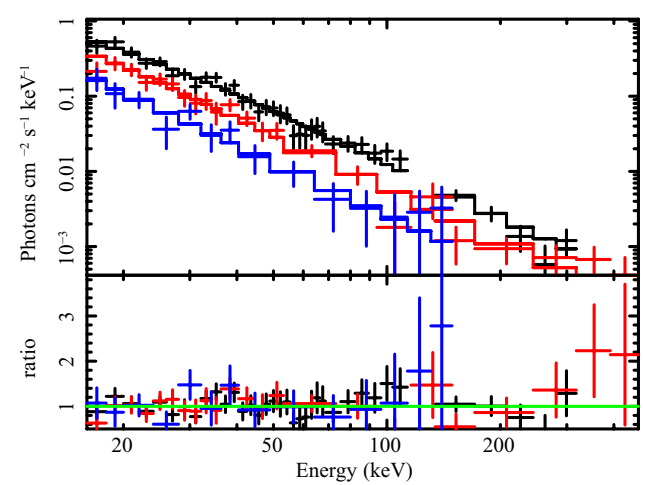

GRB 070917

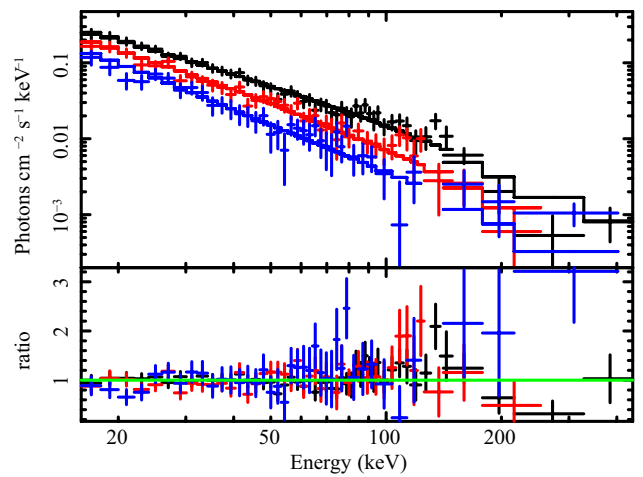

Figure 3: Time-resolved spectra and their ratio to the best-fit GRBM ratios of GRB 060117 (left) and GRB 070917 (right). The black, red and blue marks and lines indicate spectra and the model at 1s, 2s, and $3 \mathrm{~s}$ after the FRED peak of each event.

\subsection{GRB 080413B}

The time-averaged spectrum in the FRED decay phase of GRB 080413B prefers GRBM to BBPL significantly. However, the time-resolved spectra exhibit no decreasing of normalization formally, unlike the 2 GRBs reported in the previous subsection. Table 2 shows the derived time evolution of turnover energy $\left(E_{0}\right)$ for both the fast and slow cooling cases. The fast cooling case, under the assumption described in the previous subsection, shows smaller values of the turnover energy than the slow cooling ones evaluated with a constant normalization. However, the required 
Table 1: Time evolution of turn over energy $\left(E_{0}(t)\right)^{\dagger}$

\begin{tabular}{c|cc}
\hline \hline time after peak & GRB 060116 & GRB 070917 \\
\hline $1-\mathrm{s}$ & $53.4_{-4.0}^{+4.2} \mathrm{keV}$ & $195_{-22}^{+26} \mathrm{keV}$ \\
$2-\mathrm{s}$ & $29.1_{-2.9}^{+3.0} \mathrm{keV}$ & $82.8_{-8.4}^{+9.3} \mathrm{keV}$ \\
3 -s & $13.3_{-4.0}^{+2.4} \mathrm{keV}$ & $37.6_{-4.4}^{+4.6} \mathrm{keV}$ \\
$\left(\chi^{2} /\right.$ d.o.f. $)$ & $54.7 / 70$ & $109 / 127$ \\
\hline$\eta$ & $-1.13_{-0.21}^{+0.23}$ & $-1.48_{-0.25}^{+0.26}$ \\
\hline
\end{tabular}

$\dagger$ : Fast-cooling time evolution with GRBM normalization is assumed.

time index $\eta$ in the fast-cooling case is far from the expected value $(\eta=-2 / 3)$. On the other hand, in the slow cooling case, the derived time index agrees with the expected value of $\eta=-1 / 2$ if we allow the confidence level of 95\%. A similar case has been observed in GRB 100707B [2]. The obtained time index is consistent with the one expected in a slow synchrotron or inverse-Compton cooling regime, but is not conclusive, as the fast-cooling or other models are not rejected due to the limited statistics.

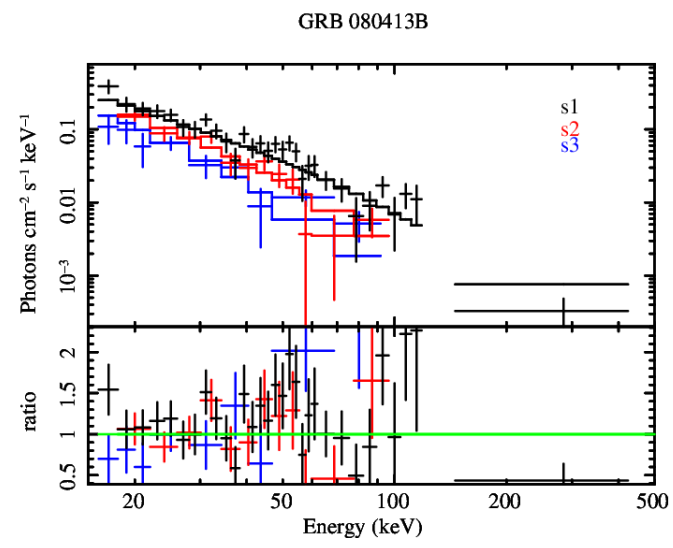

Figure 4: Time-resolved spectra of GRB 080413B in the slow-cooling regime. The employed models and color codes are the same as those in Figure 3.

\section{Discussion and conclusion}

We here discuss the physical mecahnism of those FRED-type GRBs, combining both this wide-band study on 3 GRBs and our preceding study on 7 FRED-type GRBs for the narrower energy-band with only Suzaku/WAM [2]. For all of those 10 FREDs, we found that the decay-time constants $(\tau)$ of their energy-resolved light curves are well described with power-law functions of energy $(E)$ as $\tau=E^{-0.29 \pm 0.12}$. The energy indices of 6 of 10 FREDs suggest a simple synchrotron (or inverse-Compton) cooling, whereas $\tau$ of the remaining 4 do not agree with the expected value of -0.5 with a $90 \%$ confidence level, which suggests geometrical change in radiation surface and/or a need of additional component(s). 
Table 2: Time evolution of turnover energy $\left(E_{0}(t)\right)$ of GRB 080413B

\begin{tabular}{c|cc}
\hline \hline time after peak & Fast cooling & Slow cooling $^{\dagger}$ \\
\hline $1-\mathrm{s}$ & $59.1_{-5.7}^{+6.0} \mathrm{keV}$ & $75.5_{-7.7}^{+8.0} \mathrm{keV}$ \\
$2-\mathrm{s}$ & $35.2_{-4.3}^{+4.7} \mathrm{keV}$ & $38.2_{-4.9}^{+5.4} \mathrm{keV}$ \\
3 -s & $24.2_{-3.8}^{+4.1} \mathrm{keV}$ & $23.8_{-3.7}^{+4.0} \mathrm{keV}$ \\
$\left(\chi^{2} /\right.$ d.o.f. $)$ & $57.4 / 49$ & $66.1 / 49$ \\
\hline$\eta$ & $-0.79_{-0.27}^{+0.26}$ & $-1.03_{-0.28}^{+0.27}$ \\
\hline
\end{tabular}

$\dagger$ : No decrease in normalization of the GRB model is assumed.

We also carried out time-resolved spectroscopy for the 3 GRBs presented in this paper, following the same approach as for the study of the 7 FREDs observed by WAM [2]. All 3 FRED spectra are well reproduced by the GRBM model. The behavior of 2 FREDs (GRB 060117 and GRB 070917) are consistent with those expected in the fast-cooling regime, whereas the observed data for the other one (GRB 0080413B) did not have sufficient statistics to constrain the cooling regime. These $3 \mathrm{GRBs}$ are notable to be well explained with a simple thin-plasma emission model, unlike the case of GRB 081224, which is well reproduced with a cooling blackbody [2].

\section{References}

[1] J. P. Norris, et al. Long-Lag, Wide-Pulse Gamma-Ray Bursts, ApJ 627 (2005) 324 [arXiv:astro-ph/0503383].

[2] M. S. Tashiro, et al. Spectral Evolutions in Gamma-Ray Burst Exponential Decays Observed with Suzaku WAM, PASJ 64 (2012) 26 [arXiv: 1110.4246$].$

[3] R. Sari, et al. Spectra and Light Curves of Gamma-Ray Burst Afterglows, ApJ 497, L17

[4] D. Band, et al. BATSE observations of gamma-ray burst spectra. I - Spectral diversity, ApJ 413 (1993) 281

[5] P. Mészáros, M. J. Rees, Multi-GEV Neutrinos from Internal Dissipation in Gamma-Ray Burst Fireballs, ApJ 530 (2000) 292 [arXiv: astro-ph/ 0007102 ].

[6] F. Ryde, A. Pe'er, A., Quasi-blackbody Component and Radiative Efficiency of the Prompt Emission of Gamma-ray Bursts, ApJ 702 (2009) 1211 [arXiv: 0811 . 4135]

[7] S. Guiriec, et al. Evidence for a Photospheric Component in the Prompt Emission of the Short GRB 120323A and Its Effects on the GRB Hardness-Luminosity Relation ApJ 770 (2013) 32

[8] J. M. Burgess, et al. An Observed Correlation between Thermal and Non-thermal Emission in Gamma-Ray Bursts, ApJ, 784 (2014) L43 [arXiv: 1403.0374 ].

[9] S. D. Barthelmy, et al. The Burst Alert Telescope (BAT) on the SWIFT Midex Mission, SSR, 120 (2005) 143 [arXiv:astro-ph/0507410].

[10] K. Yamaoka, et al. PASJ, 61 (2009) S35.

[11] G. B. Rybicki, A. P. Lightman, Radiative processes in astrophysics, Wiley-Interscience, New York, 1979. 